\title{
Estudio cualitativo sobre los significados de la Medicina Complementaria y Alternativa para el cáncer en estudiantes de Ciencias de la Salud*
}

\section{Qualitative Study about the Meanings of Complementary and Alternative Medicine for Cancer in Students of Health Sciences}

Recepción: 23 Septiembre 2016 | Aceptación: 30 Octubre 2017

\author{
Ximena Palacios-Espinosa \\ Universidad del Rosario, Colombia \\ ORCID: http://orcid.org/0000-0002-9503-139X \\ IRENE BARBIERI ${ }^{\mathrm{a}}$ \\ Università degli Studi di Trento, Italia \\ ORCID: http://orcid.org/0000-0002-0209-7139 \\ María Alejandra Enciso Osorio \\ Universidad del Rosario, Colombia \\ ORCID: http://orcid.org/0000-0001-9630-3257 \\ Camila Andrea Junca Olea \\ Universidad del Rosario, Colombia \\ ORCID: http://orcid.org/0000-0003-0767-860X
}

a Autor de correspondencia. Correo electrónico: irene.barbieri3@gmail.com

Para citar este artículo: Palacios-Espinosa, X., Barbieri, I., Enciso Osorio, M. A., \& Junca Olea, C. A. (2018). Estudio cualitativo sobre los significados de la Medicina Complementaria y Alternativa para el cáncer en estudiantes de Ciencias de la Salud. Universitas Psychologica, 17(4), 1-10. https:// doi.org /10.11144/Javeriana.upsy17-4.ecsm

\section{RESUMEN}

Las representaciones sociales (RS) han sido desde siempre un concepto fundamental para la psicología (Moscovici, 1973). El presente estudio cualitativo utiliza el concepto de RS aplicado al campo de la Salud, con el objetivo de analizar las RS de la Medicina Complementaria y Alternativa (MCA) para el cáncer, en estudiantes de Ciencias de la Salud, utilizando el análisis temático. Los resultados indican que las RS de la MCA se han elaborado con base en elementos muy diversos, pero donde prevale una objetivación y un anclaje de la MCA en lo que es común y familiar, más que en el conocimiento científico. Lo anterior para reflexionar sobre la formación de los futuros profesionales y sus prácticas en la cura del cáncer. Palabras clave

representaciones sociales; medicina complementaria y alternativa; cáncer; estudiantes del área de la salud; análisis cualitativo.

\footnotetext{
ABSTRACT

Social representations (SR) have always been a fundamental concept for psychology (Moscovici, 1973). This qualitative study uses the concept of SR, applied to the Health's field; the aim is to analyze the SR of Complementary and Alternative Medicine (CAM) for cancer in students of Health Sciences, using the methodology of thematic analysis. Results indicate that the SR of CAM have been developed based on many different elements, but where prevails an objectification and anchoring of CAM on what is common and familiar, rather than based on scientific knowledge. This to reflect on the training of future professionals and their practices in curing cancer.
} 
Keywords

social representations; complementary and alternative medicine; neoplasms; health occupations students; qualitative analysis.

El cáncer representa altas tasas de morbilidad y de mortalidad en el mundo, aun cuando las investigaciones en el área médica (Undreiner, 2008) han hecho grandes aportes para avanzar en la cura, especialmente a través de tratamientos centrados en cirugía, quimioterapia y radioterapia. Sin embargo, la efectividad de estos tratamientos es incierta y sus efectos secundarios pueden incentivar la búsqueda y el uso de otras opciones terapéuticas como la Medicina Complementaria y Alternativa (MCA), por parte de los pacientes. Para el cáncer, existe evidencia del uso de MCA a través de acupuntura, yoga, homeopatías, medicina china, medicina ayurveda, suplementos dietarios, mindfulness, bioenergética (Cassileth \& Deng, 2004) y algunos de mayor uso entre los pacientes como hierbas medicinales, productos minerales y suplementos vitamínicos (Hendershot, Dixon, Kono, Shin, \& Pentz, 2014). La literatura muestra que el uso de MCA tiene efectos tanto negativos como positivos. En lo que concierne a los efectos negativos se encuentran: problemas gastrointestinales, fotosensibilidad, problemas dermatológicos, alergias (Huebner, Münstedf, Mücke, \& Micke, 2012), sangrado, daño hepático (Arslan, Tural, \& Akar, 2013), interrupción del efecto de algunos fármacos sobre la actividad tumoral (Pihlak et al., 2014) y disminución del efecto de la quimioterapia y la radioterapia (Arslan et al., 2013). Con respecto a los efectos positivos, alguna evidencia muestra que la MCA puede ser eficaz para reducir el estrés y contribuir en el mejoramiento de la calidad de vida de los pacientes (Klafke, Eliot, Wittert, \& Oliver, 2012); minimizar los efectos secundarios de la quimioterapia (Smith, Clavarino, Long, \& Steadman, 2014); manejar náuseas y vómito (Huebner et al., 2012; Sánchez \& Venegas, 2010), fortalecer la competencia inmunológica (Yap, See, Kuo, Chui, \& Chan, 2012), disminuir la toxicidad gastrointestinal y genitourinaria postradiación (Aksu, Bozcuk, \& Korcum, 2008).

Recientemente, Johnson, Park, Gross y Yu (2018) reportaron que el uso de medicina alternativa entre los pacientes oncológicos puede tener implicaciones en las tasas de supervivencia. La evidencia muestra que los pacientes con cáncer recurren a alternativas diferentes a la medicina convencional (Sánchez \& Venegas, 2010; Conrad et al., 2013) y es posible que esta decisión esté basada en diversos factores como RS de los diferentes tipos de medicina, la alta aceptación de la MCA y el pensamiento mágico (Borrell, 2005).

Las RS conforman un concepto propuesto por Serge Moscovici para referirse a aquel conocimiento del sentido común, producto de la interacción y del intercambio de información dentro de los grupos sociales que suelen emerger en los momentos de crisis o de conflicto (Mora, 2002). A partir del concepto de representación social, Moscovici planteó la Teoría de las Representaciones Sociales (TRS). En palabras de Bauer y Gaskell (2008), esta teoría se constituye en un marco teórico de referencia coherente para el estudio y la comprensión de la transmisión del conocimiento en la vida cotidiana, que proviene de diversas fuentes, y revela que el pensamiento está influido por la colectividad. En este sentido, la TRS se posiciona como una herramienta para analizar la realidad social y ofrecer una explicación a los comportamientos de los individuos, teniendo en cuenta la cultura y las estructuras sociales (Herner, 2010).

Mora (2002) describe tres dimensiones de las RS: (1) la información (conocimientos con los que cuentan las personas sobre el objeto de representación), (2) el campo de representación, en el cual se organiza el contenido de la representación de manera jerarquizada y diversa y que a la vez permite precisar el objeto de representación y (3) la actitud (posición de favorabilidad o no hacia el objeto de representación).

El presente estudio se desarrolló con el objetivo de analizar las RS de la MCA en estudiantes de carreras de Ciencias de la Salud, a partir del enfoque procesual (Banchs, 2000). 
Este enfoque considera que solo un abordaje hermenéutico permite conocer las RS que las personas construyen en el mundo donde viven en la búsqueda de sentido, el análisis de los simbolismos, de los significados y del lenguaje (Banchs, 2000).

Siendo el cáncer una enfermedad tan común, es probable que la mayoría de los profesionales de la salud y en este caso, los estudiantes en formación, deban atender en algún momento pacientes con dicho diagnóstico. En este sentido, las RS pueden constituir una herramienta fundamental de análisis, con el fin de comprender sus actitudes, sus ideas y comportamientos de acuerdo al contexto y formación, pues de dichos factores depende la intervención idiográfica de los pacientes. Adicionalmente, es necesario tener en cuenta que no hay antecedentes de investigaciones sobre el tema de las RS de la MCA en este tipo de población, lo que hace el presente estudio aún más relevante para contribuir en el abordaje integral de esta enfermedad. En general, existen estudios acerca de la información y las actitudes que tienen los estudiantes y profesionales de la salud sobre dicha medicina con base en modelos teóricos diferentes al de las RS. El elemento común en estos estudios indica que tanto los estudiantes como algunos profesionales de la salud carecen de conocimientos sobre la MCA y sus distintas prácticas (Majeed et al., 2007), puesto que no es un tema que se incluya en los planes de estudio; sin embargo, manifiestan actitudes positivas hacia este tipo de medicina (Ditte, Schulz, Ernst, \& Schmid, 2010; Sierra-Ríos, Urrego-Mendoza, \& Jaime-Jaimes, 2012).

\section{Objetivos}

El objetivo general de este estudio estuvo dirigido a analizar las RS de la MCA para el cáncer, en estudiantes de carreras de Ciencias de la Salud. En consecuencia, se propusieron como objetivos específicos: (1) identificar las RS sobre la MCA para el cáncer, (2) comprender el campo de representación de MCA y (3) conocer las actitudes hacia la MCA.

| Universitas Psychologica | V. I 7 | No. 4 | 2018 |

\section{Método}

\section{Participantes}

En este estudio de tipo cualitativo descriptivo/ interpretativo (Riger \& Sigurvinsdottir, 2016), la población estuvo conformada por estudiantes universitarios de carreras de Ciencias de la Salud y se incluyeron los de una escuela de Ciencias de la Salud de una universidad privada de Bogotá, Colombia. Específicamente, se contactaron los estudiantes de las carreras de Medicina, Psicología, Fisioterapia, Terapia Ocupacional e Ingeniería Biomédica, las cuales hacen parte de esa Escuela. El muestreo fue seleccionado en bola de nieve (Noy, 2008). La muestra total fue de 121 estudiantes, divididos en 14 grupos focales. Cada grupo focal tuvo un promedio de 8 a 9 personas.

\section{Instrumentos}

Para recoger la información se condujeron grupos focales basados en una guía de preguntas que fue elaborada ad hoc a partir de los objetivos del estudio. En particular, las nueve preguntas estaban relacionadas con lo siguiente: conocimiento/información de los estudiantes sobre la MCA (p. ej., ¿Qué tratamientos de MCA ha escuchado que realizan los pacientes con cáncer?, ¿Cómo se enteró de estos tratamientos de MCA?, ¿En qué consisten esos tratamientos de MCA?); opinión acerca de la MCA (p. ej., ¿Cuál es su opinión acerca de la MCA para el cáncer?); efectos de la MCA, específicamente en la eficacia y los efectos secundarios (p. ej., ¿Para qué sirven exactamente esos tratamientos de MCA?, ¿Tiene algún riesgo hacer este tipo de tratamientos de MCA para el cáncer?, ¿Qué efectos considera usted que tiene la MCA en el tratamiento médico convencional para el cáncer?); las prácticas de uso de los tratamientos (p. ej., iHan hecho ustedes tratamientos con MCA?, ¿Por qué?, ¿Les recomendarían ustedes esos tratamientos de MCA para el cáncer a otras personas?, ¿Por qué?). Estas fueron solamente preguntas guía, pero como es esperable en el desarrollo 
de los grupos focales, surgieron otras preguntas que permitieron complementar y enriquecer la discusión.

Procedimiento de recolección de datos

Con el fin de desarrollar los grupos focales, se obtuvieron las debidas autorizaciones de la universidad para la realización de investigación con estudiantes. La participación de los estudiantes fue voluntaria. Cada uno de ellos dio su consentimiento informado antes de empezar. Los grupos focales fueron grabados y luego la información fue transcrita para proceder al análisis.

\section{Procedimiento de análisis de datos}

Para analizar los datos de los grupos focales, se utilizó la técnica del análisis temático, "un método para identificar, analizar y reportar patrones (temas) en los datos" (Braun \& Clarke, 2006, p. 79) que permite encontrar temas en una base de datos de tipo cualitativo (Riger \& Sigurvinsdottir, 2016). A partir de estos temas, es posible identificar patrones de significado. Según las indicaciones de Braun y Clarke (2006), se condujo, a mano, el análisis a través de seis fases:

(1) Familiarización con los datos (transcripción y lectura de la información).

(2) Generación de códigos iniciales de análisis. Estos códigos, básicamente palabras y frases, se crearon a partir de todo el cuerpo de datos (data corpus) recolectado en los grupos focales al hacer las preguntas mencionadas y que, por consiguiente, versaban sobre la MCA para el cáncer. Se leyó cada frase y de cada una de ellas, se extrajeron uno o varios códigos.

(3) Identificación de temas potenciales en los códigos formulados inicialmente (conjuntos de códigos se desarrollaron en temas comunes). En esta fase, algunos de los códigos creados en la fase anterior, se convirtieron en temas principales y otros se agruparon en nuevos temas relevantes para el objetivo del estudio, de tal manera que al final de esta etapa lo que se obtuvo fue un amplio conjunto de temas (categorías principales) y subtemas (categorías menores que permitieron definir y describir los temas).

(4) Revisión de los temas. Durante esta etapa, tal y como lo establecen Braun y Clarke (2006), el objetivo fue refinar los temas creados en la fase anterior, logrando un patrón coherente de información, pero a la vez una clara distinción entre los temas. Con base en esto, se creó un primer esquema que permitiera observar la coherencia entre los temas.

(5) Definición de los temas centrales y los subtemas. En esta fase, la tarea se centró en narrar, describir cada uno de los temas. Los temas recibieron un nombre que fuera lo suficientemente ilustrativo de lo que se buscaba mostrar. Se descartaron algunos de los temas que definitivamente, no lo eran. Con esta revisión, se creó el mapa temático final.

(6) Elaboración de las conclusiones, lo que dio origen al reporte de investigación.

\section{Resultados}

A través de los resultados obtenidos, se construyó un esquema de temas y subtemas relacionados que permitió identificar las RS, develar el campo de representación (Figura 1) y analizar las actitudes hacia la MCA entre los estudiantes de carreras de Ciencias de la Salud.

En las fases iniciales, se crearon aproximadamente 230 códigos y 30 temas. Como resultado del procedimiento previamente descrito, se encontraron cinco grandes ejes en los que pueden organizarse los temas y subtemas relacionados con la MCA para el cáncer y que le dan significado a este objeto de representación entre los estudiantes de Ciencias de la Salud: (1) origen; (2) naturaleza; (3) definición; (4) funciones y eficacia y (5) fuente de conocimiento. Como puede observarse en la Figura 1, el origen de la MCA se explicó a través de dos temas, origen en la tradición y en el conocimiento científico, a partir de los cuales se encontraron cuatro subtemas relacionados. La definición se explicó también con dos temas, conjunto de prácticas diferentes/pseudociencia y pensamiento mágico, de los cuales se derivaron 
ocho subtemas. La naturaleza de la MCA se explicó a través de dos temas, natural e inofensiva y tóxico y riesgosa, de los cuales se encontraron ocho subtemas. Las funciones y eficacia de la MCA, se explicó con tres temas, función curativa, función preventiva y función paliativa, de los cuales se derivaron cuatro subtemas y de estos siete subtemas más. Finalmente, la fuente de conocimiento sobre MCA se explicó con cinco temas: fuentes de conocimiento terciarias y de predominio no científico, elección depende del nivel educativo, elección depende del bajo costo, elección depende del estadio del cáncer y la elección depende de experiencias de seres cercanos, de los cuales derivaron cuatro subtemas. Las actitudes, componente afectivo de las RS y que determina la posición de los estudiantes frente a la MCA para el cáncer, se analizaron de manera transversal a todos los temas que emergieron, extrayendo la valencia positiva o negativa otorgada por los estudiantes a la MCA.

Según los estudiantes de Ciencias de la Salud, la MCA se representa como un conjunto de prácticas diferentes y pseudocientíficas, entre las cuales parece predominante la idea de la MCA como indiferenciada de la medicina popular (o la consideración de los dos tipos de medicina dentro de la misma categoría). En particular, los participantes de la carrera de Medicina asocian la MCA con un conjunto de prácticas de carácter pseudocientífico que no cuentan con respaldo teórico suficiente, mientras que los estudiantes de Ciencias de la Rehabilitación, Psicología e Ingeniería Biomédica consideran que se respalda en un marco científico que cuenta con evidencia cada vez más rigurosa. Las prácticas que más asocian con dicha medicina son la homeopatía, la acupuntura y la bioenergética. Los estudiantes también definen la MCA como algo asociado al pensamiento mágico de varios tipos: $\mathrm{MCA}$ relacionada con la energía, la charlatanería, la brujería, la magia y la fe.

Adicionalmente, en relación con su naturaleza, la MCA es representada de forma ambivalente como un tipo de medicina natural o inofensiva, pero que a su vez puede también ser tóxica o de riesgo. De acuerdo con los estudiantes, la naturaleza tóxica se relaciona con los efectos negativos de las prácticas de MCA.

\section{Figura 1}

Esquema de temas y subtemas emergentes.

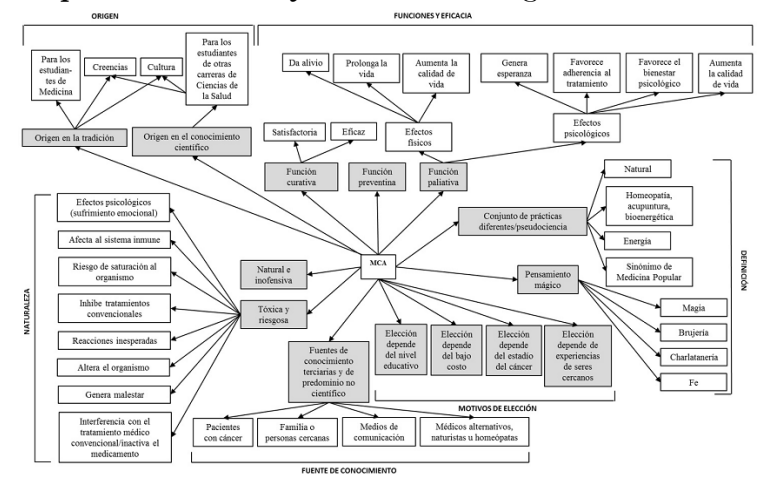

Nota. Los temas se encuentran en las casillas de color gris, mientras los subtemas en las casillas de color blanco.

Entre los efectos negativos más destacados están: la afectación del sistema inmune, los efectos psicológicos referidos al sufrimiento emocional y la interferencia con el tratamiento médico convencional que a la vez genera malestar, altera el organismo, provoca reacciones contrarias, inactiva el medicamento, genera riesgo por la saturación y la inhibición de ambos tratamientos. También su origen se representa a través de dos temas en contraste: las tradiciones, las creencias populares y la cultura o a través del conocimiento científico.

Con respecto a la función de la MCA y su consecuente eficacia para el tratamiento del cáncer, las RS de los estudiantes se centran en tres temas importantes: MCA como tratamiento con función curativa, preventiva y paliativa. En este sentido, de acuerdo con los estudiantes, las prácticas pertenecientes a la MCA son inagotables y además, cuentan con varios objetivos como complementar, curar, paliar, prolongar la vida, prevenir el cáncer y favorecer el bienestar psicológico, puesto que conciben al ser humano como integral. Cabe destacar que los estudiantes consideran que los principales objetivos de la MCA son paliar y complementar. En particular, la función paliativa parece de gran relevancia: es un tratamiento que tiene efectos tanto físicos (alivia, prolonga la vida, aumenta la calidad de vida) como psicológicos (genera 
esperanza, favorece adherencia al tratamiento, favorece el bienestar psicológico, aumenta la calidad de vida).

La forma en que los estudiantes construyen las representaciones sobre la MCA, se relaciona con el medio a partir del cual adquieren la información sobre dicho tema. Su fuente de conocimiento es de tipo terciario (no hay una búsqueda de información en primera persona) y prevalentemente no científico-académica. En efecto, reportaron haber aprendido sobre MCA a través de pacientes con cáncer, familia o personas cercanas, medios de comunicación y en parte por médicos alternativos, naturistas u homeópatas.

Entre los factores que los estudiantes asocian con la elección de la MCA están: el nivel educativo, las experiencias de seres cercanos y el estadio de la enfermedad, ya que consideran que en la fase inicial y terminal es más probable que se haga uso de la MCA: "Depende del cáncer que uno tenga o el grado" (GF1, P4). Así mismo, las prácticas de MCA son consideradas por los estudiantes como más económicas y más asequibles: "La gente lo usa porque son más accesibles, porque no creo que pueda pagar una quimioterapia" (GF3, P8).

En relación con las actitudes hacia la MCA, se pudo observar entre los estudiantes una cierta ambigüedad aunque las actitudes positivas parecen ser prevalentes respecto a las negativas: "Yo pienso que también esos métodos, uno tiene que ser consciente de que pueden servir o no pueden servir, pero uno tiene que poner la mayor fe, esperanza, lo que sea, en ese aspecto" (GF2, P1). A partir de los temas encontrados, se identificaron actitudes positivas en relación con varios beneficios de la MCA desde diversos aspectos, que a pesar de que carecen de información científica para ellos, favorecen que su postura se incline hacia la aceptación de la MCA.

\section{Discusión}

Los resultados del presente estudio indican que las RS de la MCA se han elaborado con base en elementos muy diversos, lo que se evidencia en contradicciones como origen científico vs. pseudocientífico, inofensiva vs. tóxica, eficacia vs. charlatanería, función paliativa vs. curativa. Se evidencian además temas fundamentales de la representación como la inagotabilidad de opciones terapéuticas y la indiferenciación con la medicina popular. En el campo representacional, se encuentran actitudes prevalentemente positivas hacia la MCA. Los resultados permiten reflexionar sobre la necesidad de incluir en los planes de estudio de carreras de Ciencias de la Salud, temas sobre otros tipos de alternativas terapéuticas, que a la vez pueden contribuir a mejorar la preparación para su práctica profesional. De acuerdo con los resultados del presente estudio, es posible concluir que los estudiantes no diferencian entre la medicina popular y la MCA, lo cual es coherente con el hallazgo de Majeed et al. (2007), según el cual los estudiantes no reconocen con claridad las prácticas pertenecientes a los diversos tipos de medicina. Es posible que a esto contribuya el hecho de que actualmente, en el sistema de salud colombiano, la MCA no goza de estatus científico y en consecuencia, la clasificación existente de sus prácticas continúa siendo diversa.

La asociación que hacen los estudiantes de la MCA con la energía, la brujería, la charlatanería, la magia y la fe, puede entenderse con base en el planteamiento de Borrell (2005) sobre la interpretación de la enfermedad mediante el pensamiento mágico que, en este caso, permite vincular las creencias sobre las energías, la fe y la motivación para vivir con las ideas que surgen en los estudiantes acerca de los factores que influyen en el afrontamiento de la enfermedad (p. ej., la voluntad o la fe que los pacientes depositen en su proceso).

Tradiciones, creencias, cultura y conocimiento científico fueron identificados como los factores de origen de la MCA. Sin embargo, se encontró una diferencia entre los estudiantes con respecto al sustento científico de la MCA. Los estudiantes de medicina consideran la MCA carente de respaldo teórico. ¿Es posible que esto esté particularmente asociado con el hecho de que en Colombia los planes de estudio de la 
carrera de Medicina estén centrados en formar médicos alópatas, con competencias basadas en la medicina convencional? Por otra parte, los estudiantes de Ciencias de la Rehabilitación, Psicología e Ingeniería Biomédica, consideraron que la MCA tiene respaldo científico y por tanto mostraron actitudes más positivas hacia ella. Este hallazgo es coherente con el de Sierra et al. (2012), según el cual los estudiantes que participaron en su estudio consideraron que la MCA tiene bases científicas demostrables y que, adicionalmente, tiene un carácter inagotable con respecto al de la medicina convencional, donde las opciones no superan la cirugía, radioterapia y quimioterapia; específicamente, como lo plantean Barnes, Bloom y Nahim (2008), consideran que tiene diversos objetivos como prevenir y tratar, pero le otorgaron además efectos fundamentalmente paliativos.

Los estudiantes tienen varias representaciones sobre los factores asociados con la elección de la MCA. En primer lugar, la conciben como más económica, sin embargo, dicha idea es errónea; esto podría estar asociado con la confusión conceptual entre la medicina popular y la MCA. En segundo lugar, otros de los factores asociados por los estudiantes con la elección de la MCA son el nivel educativo, las experiencias de seres cercanos y el estadio de la enfermedad. Es importante destacar que el componente más influyente en las ideas sobre el uso de la MCA se relaciona con el pensamiento popular, que prevalece por encima de la formación académica y que permite que un individuo se adhiera a este tipo de medicina por la experiencia de otros o las concepciones de la sociedad. Con respecto a estas fuentes de conocimiento, cabe reflexionar sobre el carácter informal de las mismas debido a que la información proviene principalmente de personas con un significado afectivo (pacientes con cáncer, familia y personas cercanas), medios de comunicación masiva y de médicos que los estudiantes consideran "alternativos, naturistas u homeópatas" pero de quienes no hay evidencia de su formación.

Con respecto a las actitudes positivas de los estudiantes hacia la MCA, es posible afirmar que además de que reconocen varios beneficios de dicha medicina, la experiencia y el uso de esta, favorecen que tiendan a generalizar sus efectos deseables, incluso para una enfermedad como el cáncer, a pesar de que reconocen que no cuentan con suficiente conocimiento en este tema. Dichos resultados también se obtuvieron en el estudio realizado por Ditte et al. (2010).

A partir de los resultados obtenidos, se puede concluir que los estudiantes que participaron en este estudio tienen RS de la MCA para el cáncer, resultado de la interrelación entre aspectos psicológicos y sociales. Entre los primeros, fueron evidentes afecto, emociones, experiencias personales y entre los segundos, el contexto: un contexto universitario donde estudian carreras de Ciencias de la Salud en las que se valida casi que exclusivamente el conocimiento científico. Y además un contexto colombiano, en el que el pensamiento mágico es aceptado socialmente. Entonces, la MCA para el cáncer que en algún momento pudo ser un concepto abstracto, fue objetivada por los estudiantes materializando sus ideas, convirtiéndola en un marco cognoscitivo estable, que consideran "válido" y a partir del cual emiten opiniones, categorizan a las personas y sus acciones, y plantean posturas vinculadas a su historia personal, a los valores personales, familiares y al afecto.

En este proceso de objetivación la MCA para el cáncer, inicialmente intangible, se hace tangible, concreto y asequible al sentido común. Tal y como lo plantea la TRS, lo abstracto se hace concreto (Mora, 2002) y ha sido anclado por los estudiantes en lo que les resulta más "común" y "familiar": la medicina popular. Así, la MCA para el cáncer modifica su estatus de "inalcanzable" o "incomprensible" y se convierte en un concepto compartido con otros al vinculársele a lo que es conocido para todos: lo popular y lo práctico.

Dada la evidencia del uso de la MCA entre los pacientes con cáncer, parece necesario informar a los estudiantes de carreras de Ciencias de la Salud sobre este aspecto, dotándolos de elementos para elaborar ideas, opiniones y actitudes fundamentadas en el conocimiento formal y no en el sentido común, con el propósito de que esto revierta en una posición profesional hacia las prácticas de los pacientes con cáncer y 
en una atención que implique la comprensión de la conducta de los pacientes frente al tratamiento oncológico y durante el proceso de enfermedad.

Este estudio es tan solo una aproximación a un fenómeno que carece de sustento empírico suficiente hasta el momento. Si bien el alcance del mismo es el análisis de las RS de la MCA entre los estudiantes de carreras de Ciencias de la Salud, se espera que los resultados se constituyan en un insumo para pensar e incluso proponer estrategias educativas en estas carreras.

Aunque la naturaleza del presente estudio no permite generalizar los resultados, los participantes fueron de diferentes carreras de las Ciencias de la Salud y los resultados, muy homogéneos. Por lo tanto, se considera que este trabajo puede contribuir a poner en evidencia el escaso conocimiento que tienen los estudiantes de estas carreras tanto sobre el cáncer, cuya prevalencia a nivel mundial es muy alta, como sobre la MCA e incluso, la confusión al respecto. Pero además, su valor está también en que permite observar el significado que esta medicina tiene para ellos, descrita previamente, y esto es relevante a la hora de tomar decisiones y asumir posiciones. Por lo tanto, se propone que los programas de formación de pregrado en Ciencias de la Salud, contemplen como una de las competencias que se debe desarrollar en sus estudiantes, la identificación y comprensión de prácticas terapéuticas alternativas y complementarias; la comprensión de los aspectos biológicos, psicológicos y socioculturales vinculados y el reconocimiento del uso de las mismas como una realidad del paciente oncológico, entre muchos otros. Reconocerla como un tema u objeto de estudio, haría que la MCA y sus prácticas se conocieran, así como sus efectos secundarios y sobre la enfermedad o los síntomas. De esa manera, aumentaría el cuerpo de conocimientos y evidencia sobre la misma. Diferentes profesionales de la salud, no exclusivamente médicos, realizan prácticas de MCA. Es probable que esto esté fundamentado en una credibilidad adquirida a priori, basada en las RS de la MCA como inofensiva o natural, y no en el conocimiento. Si los estudiantes de Ciencias de la Salud recibieran formación de pregrado en estos temas, también podrían tomar decisiones informadas respecto de su práctica y formación posgradual. En consecuencia, la actitud frente a la MCA sería más profesional y menos personal.

Por último, es de anotar que el presente estudio tiene algunas limitaciones; una de ellas, que los participantes fueron todos de la misma institución y que la naturaleza del estudio no permite la generalización de los datos.

\section{Agradecimientos}

Las autoras agradecen a David Fernando Vargas Sarmiento, por su valiosa contribución en la recolección de la información y el análisis inicial que dio lugar a este artículo.

\section{Referencias}

Aksu, M. G., Bozcuk, H. S., \& Korcum, A. F. (2008). Effect of complementary and alternative medicine during radiotherapy on radiation toxicity. Supportive Care in Cancer, 16(4), 415-419. https://doi.org/10. 1007/s00520-007-0390-0

Arslan, D., Tural, D., \& Akar, E. (2013). herbal administration and interaction of cancer treatment. Journal of Palliative Medicine, 16(11), 1466-1473. https://doi.org/10.1089 /jpm.2013.0126

Banchs, M. (2000). Aproximaciones procesuales y estructurales al estudio de las representaciones sociales. Papers on Social Representations, 9, 3.1-3.15. Recuperado de http://www.psych.lse.ac.uk/psr/PSR200 0/9 3Banch.pdf

Barnes, P., Bloom, B., \& Nahin, R. (2008). Complementary and alternative medicine use among adults and children: United States, 2007. National Health Statistics Reports, 12, 1-24. Recuperado de https://www.nccam.nih.gov/sites/nccam .nih.gov/files/news/nhsr12.pdf

Bauer, M. W., \& Gaskell, G. (2008). Social representations theory: A progressive research programme for social psychology. 
Journal for the Theory of Social Behaviour, 38(4), 335-353. https://doi.org/10.1111/j.1 468-5914.2008.00374.x

Braun, V., \& Clarke, V. (2006). Using thematic analysis in psychology. Qualitative Research in Psychology, 3(2), 77-101. https://doi.org/ 10.1191/1478088706qp063oa

Borrell, F. (2005) Medicinas complementarias y alternativas: algunas reflexiones desde la bioética. Atención Primaria, 35(6), 311-313. https://doi.org/10.1157/13073418

Cassileth, B., \& Deng, G. (2004). Complementary and alternative therapies for cancer. The Oncologist, 9, 80-89. https:/ /doi.org/10.1634/theoncologist.9-1-80

Conrad, C., Muenstedt, K., Micke, O., Prott, F., Gronau, T., Muecke, R., ... Huebner, J. (2013). Survey of members of the German Society for Palliative Medicine regarding their attitudes toward complementary and alternative medicine for cancer patients. Journal of Palliative Medicine, 16 (8), 1229-1237. https://doi.org/10.1089/jpm.20 13.0084

Ditte, D., Schulza, W., Ernstb, G., \& Schmid, G. (2010). Attitudes towards complementary and alternative medicine among medical and psychology students. Psychology, Health Eु Medicine, 16(2), 225-237. https://doi.org /10.1080/13548506.2010.532559

Hendershot, K., Dixon, M., Kono, S., Shin, D., \& Pentz, R .(2014). Patients' perceptions of complementary and alternative medicine in head and neck cancer: A qualitative, pilot study with clinical implications. Complementary Therapies in Clinical Practice, 20(4), 213-218. https://doi.org/10. 1016/j.ctcp.2013.10.005

Herner, M. (2010). La teoría de las representaciones sociales: un acercamiento desde la geografía. Huellas, 14, 150-162. Recuperado de http://www.biblioteca.unlpam.edu.ar/pu bpdf/huellas/n14a08herner.pdf

Huebner, J., Münstedf, K., Mücke, R., \& Micke, O. (2012). Is there level I evidence for complementary and alternative medicine (CAM) in oncology? An analysis of
Cochrane reviews. Trace Elements and Electrolytes, 30, 29-34. https://doi.org/10.54 14/TE0X1269

Johnson, S. B., Park, H. S., Gross, C. P., \& Yu, J. B. (2018). Use of alternative medicine for cancer and its impact on survival. Journal of the National Cancer Institute, 110(1), 1-4. h ttps://oi.org/10.1093/jnci/djx145

Klafke, N., Eliott, J. A., Wittert, G. A., \& Olver, I. N. (2011). Prevalence and predictors of complementary and alternative medicine (CAM) use by men in Australian cancer outpatient services. Annals of Oncology, 23, 1571-1578. https://doi.org/10.1093/annonc /mdr521

Majeed, K., Mahmud, H., Khawaja, H., Mansoor, S., Masood, S., \& Khimani, F. (2007). Complementary and alternative medicine: perceptions of medical students from Pakistan. Medical Education Online, 12. https://doi.org/10.3402/meo.v12i.4469

Mora, M. (2002). La teoría de las representaciones sociales de Serge Moscovici. Athenea Digital, 2, 1-25. Recuperado de http://www.raco.cat/index.php/Athenea /article/viewFile/34106/33945

Moscovici, S. (1973). [Introduction]. En C. Herzlich (Ed.), Health and illness: A social psychological analysis (pp. ix-xiv). Londres: Academic Press.

Noy, C. (2008). Sampling knowledge: The hermeneutics of snowball sampling in qualitative research. International Journal of Social Research Methodology, 11(4), 327-344. https://doi.org/10.1080/13645570 701401305

Pihlak, R., Liivand, R., Trelin, O., Neissar, H., Peterson, I., Kivistik, S., ... Jaal, J. (2014). Complementary medicine use among cancer patients receiving radiotherapy and chemotherapy: Methods, sources of information and the need for counselling. European Journal of Cancer Care, 23, 249-254. https://doi.org/10.1111/ecc.1213 2

Riger, S., \& Sigurvinsdottir, R. (2016). Thematic analysis. En L. A. Jason \& 
D. S. Glenwick (Eds.), Handbook of methodological approaches to communitybased research: Qualitative, quantitative, and mixed methods (pp. 33-41). Nueva York: Oxford University Press.

Sánchez, R., \& Venegas, M. (2010). Aproximaciones complementarias y alternativas al cuidado de la salud en el Instituto Nacional de Cancerología: estudio de prevalencia. Revista Colombiana de Cancerología, 14(3), 135-143. https://doi .org/10.1016/S0123-9015(10)70094-9

Sierra-Ríos, S., Urrego-Mendoza, D., \& JaimeJaimes, J. (2012). Conocimientos, actitudes y prácticas acerca de la medicina alternativa en médicos vinculados a hospitales públicos de Cundinamarca, Colombia. Revista de Salud Pública, 14(3), 478-490. Recuperado de http://www.revistas.unal.edu.co/index.p hp/revsaludpublica/article/view/24915/402 67

Smith, P., Clavarino, A., Long, J., \& Steadman, K. (2014). Why do some cancer patients receiving chemotherapy choose to take complementary and alternative medicines and what are the risk? Asia-Pacific Journal of Clinical Oncology, 10, 1-10. https://doi.org/ 10.111/ajco.12115

Undreiner, E. (2008). Medicina convencional y complementaria vs. medicina alternativa en el tratamiento del cáncer. Banco de Drogas Antineoplásicas, 15-18. Recuperado de http://fundacionbadan.org/30temaspdf/f asc-nro5.pdf

Yap, K., See, C., Kuo, E., Chui, W., \& Chan, A. (2012). Utilizing mobile networks for the detection of clinically relevant interactions between chemotherapy regimens and complementary and alternative medicines.

The Journal of Alternative and Complementary Medicine, 18(2), 165-174. h ttps://doi.org/10.1089/acm.2010.0846

\section{Notas}

* Artículo de investigación. Este estudio hace parte de un proyecto de investigación más amplio titulado "Representaciones Sociales de la Medicina Popular, la Medicina Complementaria y Alternativa (MCA) y el Tratamiento Oncológico Convencional", que tiene como objetivo analizar las RS sobre la Medicina Popular, la MCA y el tratamiento oncológico convencional en pacientes oncológicos adultos, familiares, personal de salud, estudiantes del área de la salud y población general. La investigación recibió financiación interna de la Universidad del Rosario, a través del tiempo de los investigadores. 